\title{
ASPECTS CONCERNING THE MEASUREMENT OF FLATNESS DEVIATIONS
}

\section{Laurentiu Rece $^{1}$, Tone Ionescu ${ }^{2}$, Virgil Florescu ${ }^{3}$, Amelitta Legendi ${ }^{4}$, Carmen Ardelean ${ }^{5}$}

Abstract: This article points out the problems resulting from the calculation of flatness deviations against the adjacent surface defined according to SR EN 1101-2017 and proposes the use of a larger number of measurement points for improved accuracy, along with the identification of a reference plane surface with the help of matrix calculations. All deviations should be re-calculated by comparison to this reference plane. In order to reduce the large number of errors resulting from many rounding applied, this paper proposes the method of deviation measurement at symmetrical coordinates, while the calculation of the reference plane surface would be made with the help of an original method, whereby the number of operations is reduced to a minimum in order to increase accuracy.

UDC Classification: 621.7, DOI: 10.12955/cbup.v7.1489

Keywords: flatness deviations, accuracy, reference plane surface, MATLAB matrix.

\section{Introduction}

There are many types of component parts for which flatness deviations are very important, in view of the appropriate operation of the subassembly they belong to. For instance, many hydraulic components have one or more flat surfaces, while dispensers or hydraulic blocks have a rectangle parallelepiped shape. For instance, in the case of a hydraulic dispenser (Figure 1) the contact surface A must comply with strict flatness requirements in order to be fully operational. The task requires a measurement of the flatness deviations of surface A, along with a suggested method for the measurement of these deviations (Rece, 1999).

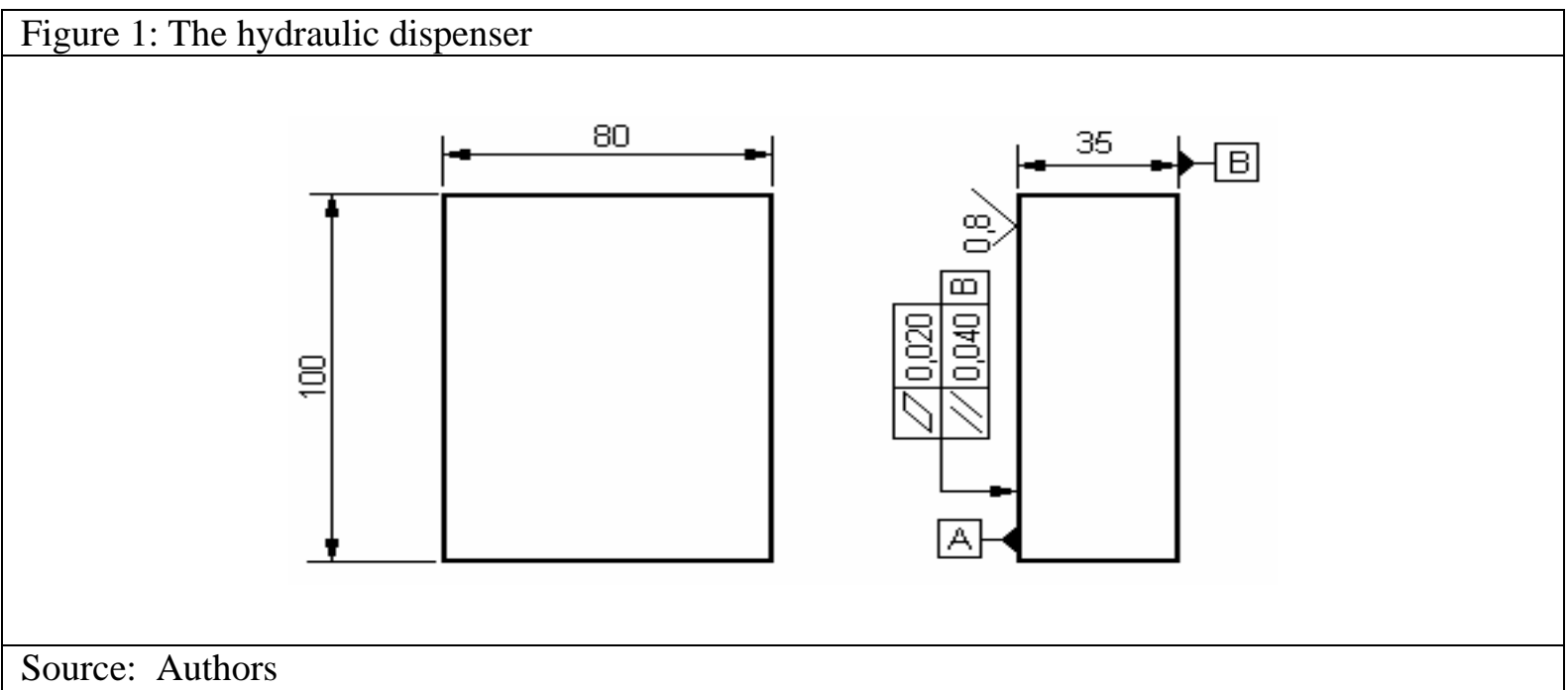

Also, the quality control of manufactured equipment imposes high accuracy requirements and, in certain cases, the flatness deviations are the most important characteristic in the view of parts' accuracy.

According to SR EN ISO 1101-2017, the flatness deviation is defined as the maximum distance between the real surface and the adjacent plane surface, measured within the limits of the reference surface or of the entire surface. The adjacent plane surface is defined as being tangent to the real surface, and thus placed so that the maximum distance between the real and plane surfaces is kept to a minimum. This definition may become a source of confusion, due to the fact that determining the adjacent plane surface is difficult, debatable or even impossible, depending on the chosen measurement method (Capitanu, Florescu \& Badita, 2016).

In practice, different methods (Legendi, A.,1994) for determining a reference plane surface are used:

\footnotetext{
${ }^{1}$ Technical University of Civil Engineering Bucharest, Romania, rece@utcb.ro

2 Technical University of Civil Engineering Bucharest, Romania, itone@ utcb.ro

${ }^{3}$ Technical University of Civil Engineering Bucharest, Romania, florescuvirgil@yahoo.com

${ }^{4}$ Technical University of Civil Engineering Bucharest, Romania, amelitta.legendi@gmail.com

5 Technical University of Civil Engineering Bucharest, Romania, c.ardelean.sti@gmail.com
} 
i. Method of the plane surface defined by three random points;

ii. Method of the plane surface defined by matrix material (MATLAB).

Apart from these two, we propose a new method, with a significantly higher degree of accuracy:

Method of the plane surface defined by the smallest squares theory/ LEAST-SQUARES METHOD.

\section{Method of the plane surface defined by three random points}

For this method, the part under study must be supported by means of three adjustable supports on a control table (Figure 2). Using a comparator clock, the points $\mathrm{O}_{1}, \mathrm{O}_{2}$ and $\mathrm{O}_{3}$ opposite the supports $\left(\mathrm{R}_{1}\right.$, $\mathrm{R}_{2}, \mathrm{R}_{3}$ ) are bent and these supports are adjusted until no deviations occur. The reference plan, parallel to the control table, will be defined by the three $\mathrm{O}_{1}, \mathrm{O}_{2}$ and $\mathrm{O}_{3}$ points. The flatness deviations will be defined as the distances measured between the points of the track surface and this reference plane surface.

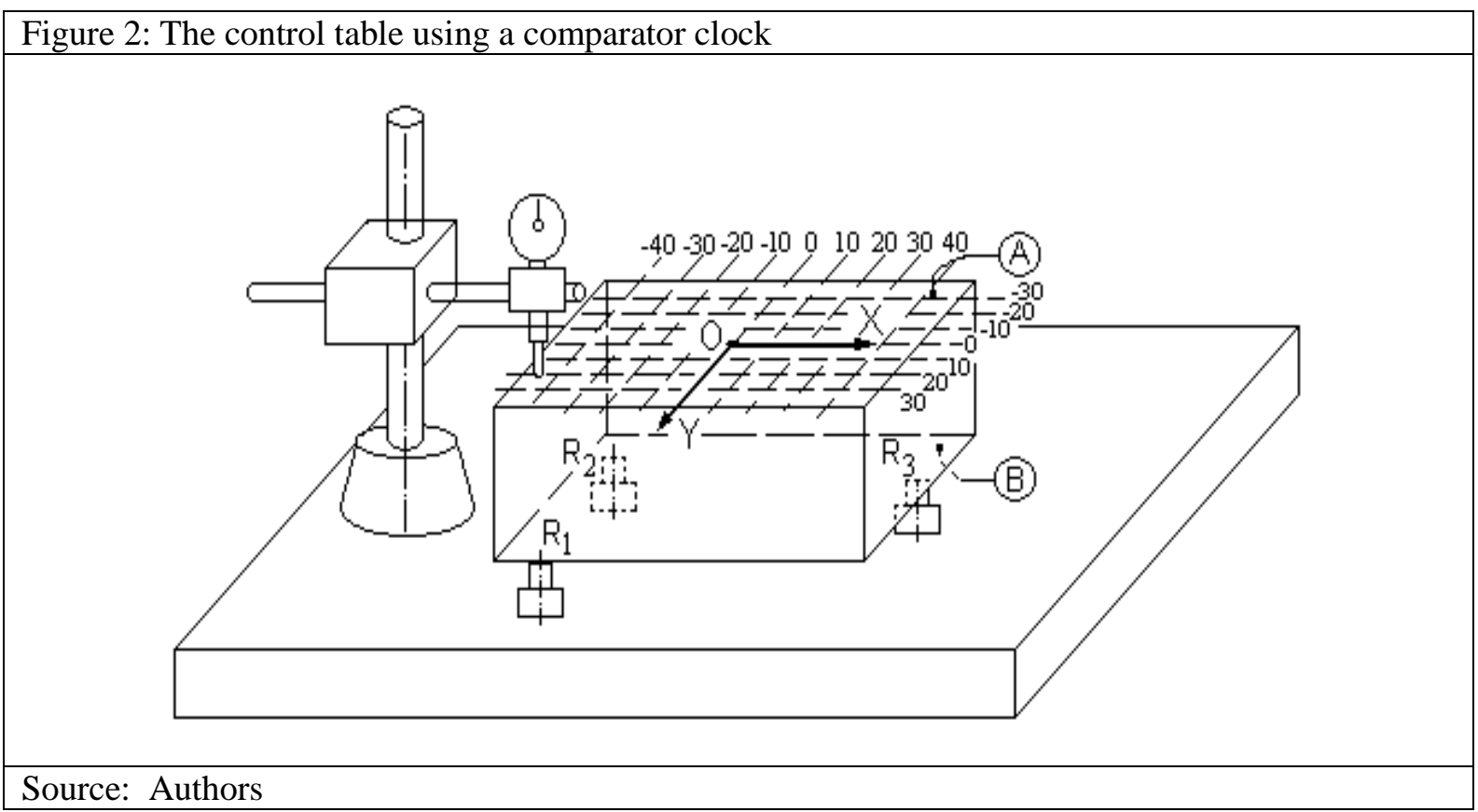

Specifically, in the case of the part under study, measurements were taken at the points of a rectangular grid of $9 \times 7=63$ points (corresponding to the Ox and Oy axes respectively), resulting in the following deviations (Table 1):

Table 1: Measurements taken at the points of the rectangular grid
\begin{tabular}{|c|c|c|c|c|c|c|c|c|c|c||}
\hline $\begin{array}{c}x \rightarrow \\
y \downarrow\end{array}$ & -40 & -30 & -20 & -10 & 0 & 10 & 20 & 30 & 40 \\
\hline-30 & 0 & 3 & 6 & 4 & 5 & 7 & 9 & 8 & 7 \\
\hline-20 & -1 & 4 & 3 & 7 & 9 & 7 & 10 & 7 & 8 \\
\hline-10 & -4 & 6 & 9 & 8 & 9 & 8 & 9 & 7 & 9 \\
\hline 0 & 0 & -2 & 5 & 9 & 8 & 9 & 9 & 7 & 0 \\
\hline 10 & -2 & 7 & 9 & 10 & 11 & $\mathbf{1 2}$ & 10 & 9 & 9 \\
\hline 20 & -3 & $-\mathbf{5}$ & 8 & 12 & 13 & 11 & 10 & 10 & 7 \\
\hline 30 & 0 & 5 & 9 & 12 & 11 & 8 & 9 & 10 & 8 \\
\hline
\end{tabular}

\section{Source: Authors}

A quick analysis of these data shows that the maximum flatness deviation, defined as the difference between the $\mathrm{max} / \mathrm{min}$ deviations, is (according to the 63-100 $\mathrm{mm}$ range and to the precision class VIII in SR EN ISO 1101-2017):

$\mathrm{AFp},{ }_{1}=13-(-5)=18 \mu \mathrm{m}<\mathrm{Tfp}=20 \mu \mathrm{m}$ 


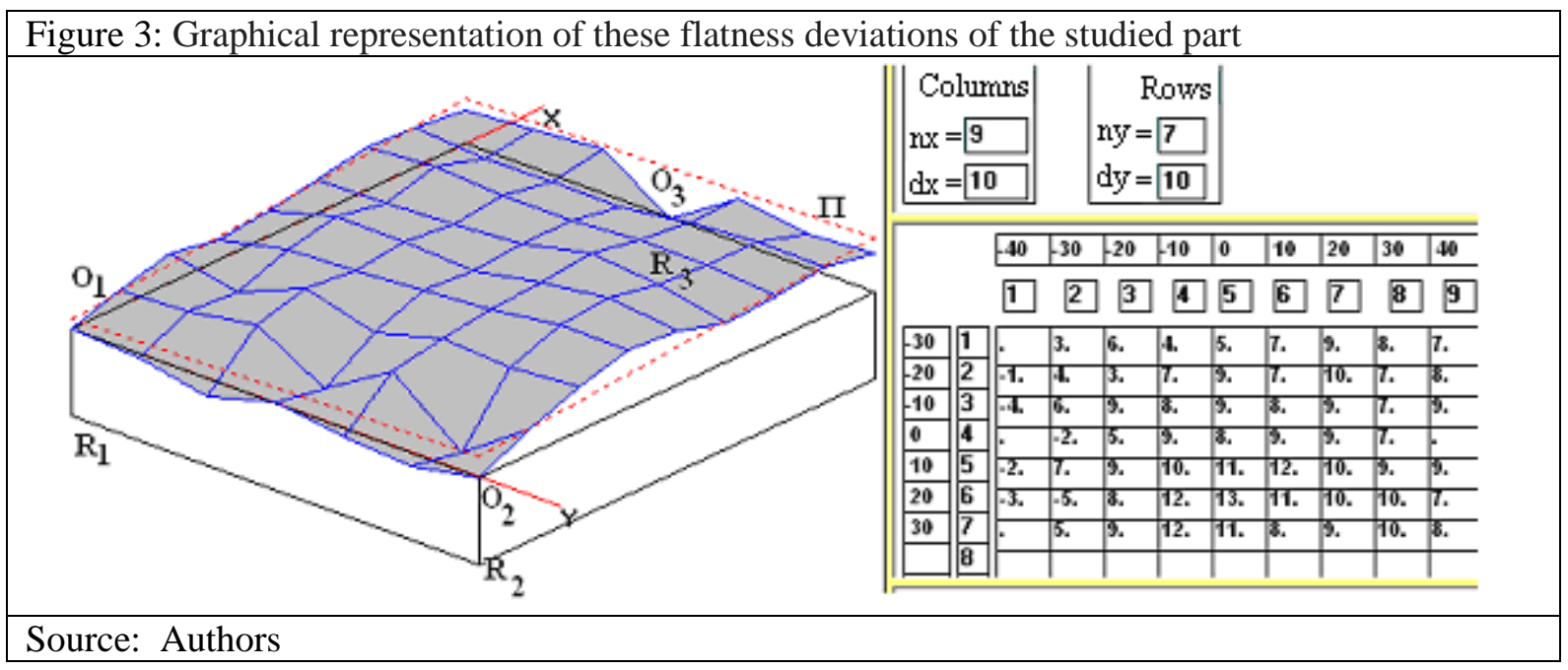

The Figure above represents flatness deviations of the studied part (performed with the "Metrology General Metrology" - Burget Jean Michel - Bayonne program), the following elements being noted:

$\mathrm{R} 1, \mathrm{R} 2$ and $\mathrm{R} 3$ are the points of contact with the three adjustable supports;

In $\mathrm{O}_{1}, \mathrm{O}_{2}$ and $\mathrm{O}_{3}$ the deviations measured are null, $\mathrm{O}_{1} \mathrm{O}_{2} \mathrm{O}_{3}$ being the first reference plane;

The right-side screen shows the data inclusion method (the number of measurement points on axes $\mathrm{O}_{\mathrm{x}}$ and $\mathrm{O}_{\mathrm{y}}$, the coordinates' spacing, the symmetric framing coordinates and the deviation values).

For this measurement method to be applied more correctly and to improve the repeatability of the measurements, the drawing should be rebuilt according to the baseline recommendations in SR EN ISO 1101-2017 (Figure 4).

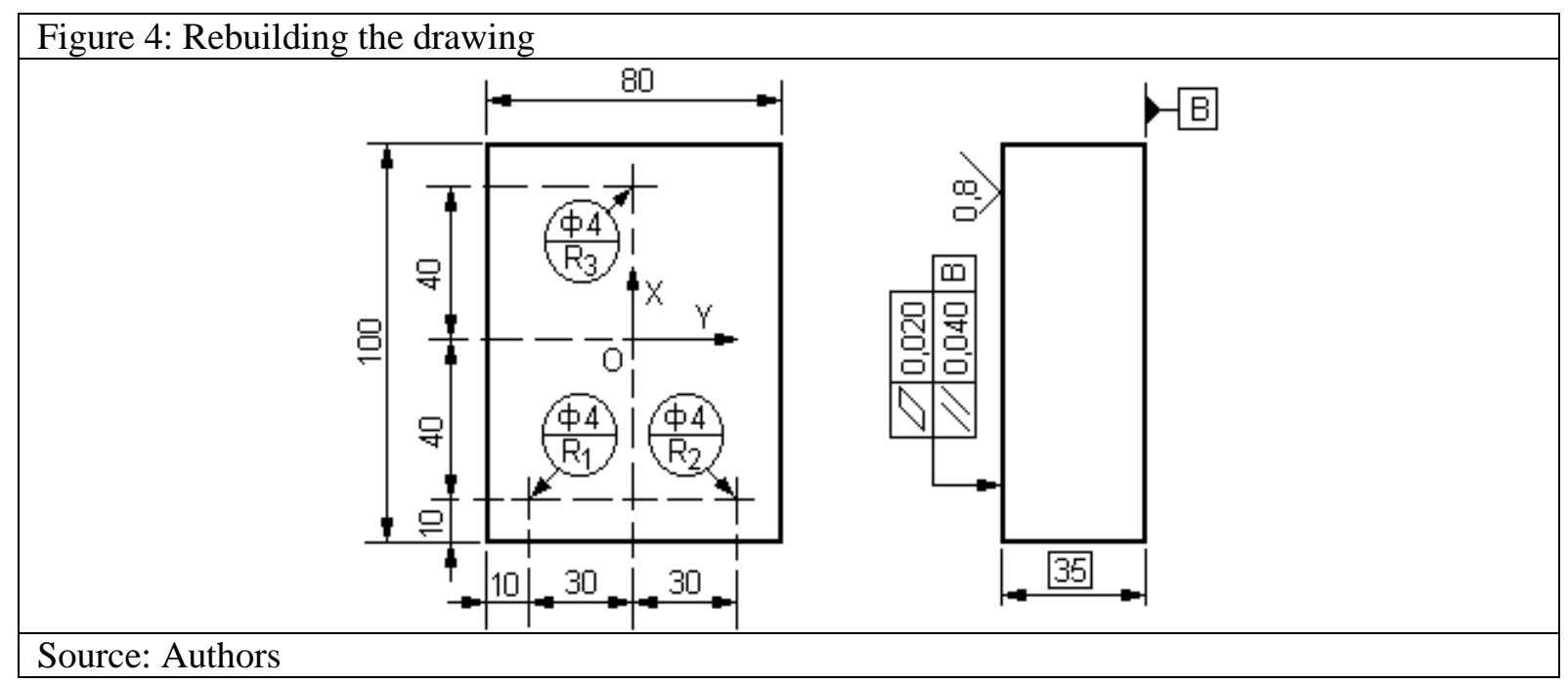

\section{Method of the plane surface defined by matrix material (MATLAB)}

For the determination of the reference plane through this method, it is necessary to start by measuring the shape deviation, using the three-random-points method. Since a large number of deviations have been determined, a system of equations with 3 unknowns, i.e. triplets ( $a, b, c)$, and a number of equations $(\mathrm{z}=\mathrm{ax}+$ by $\mathrm{c})$ equal to the number of deviations measured must be solved.

The calculation of the reference plane will be mathematically accomplished by solving a linear system with a much larger number of equations than the number of unknowns. Linear equation systems for which the number of equations is smaller than the number of unknowns can be solved through several methods: the successive substitution method, the statistical solutions method, the method of the smallest squares, and so on; however, these methods are laborious and difficult to apply in the case of systems with a large number of equations. Such a system of equations is solved in terms of 
calculations by many algebraic operations of multiplication, division, addition and decrease, which lead to a less accurate result due to the errors produced by the often-repeated rounding applied.

For fast calculation purposes, the present paper proposes the matrix solution offered by the MATLAB program, a pleasingly efficient and simple program for solving systems with more equations than unknowns. In order to reach the solution, take the data set in Table 1, which can be interpreted as the height $(\mathrm{z})$ coordinates of a plane, in the following equation:

$\mathrm{z}=\mathrm{ax}+\mathrm{by}+\mathrm{c}$

This equation can be written for all $\mathrm{n}=7 \times 9=63$ coordinate combinations (x, y), thus forming a system with 63 equations $(\mathrm{Eq})$ and 3 unknowns $(\mathrm{a}, \mathrm{b}, \mathrm{c})$, as follows:

$\begin{array}{ll}E q 1 & -40 \cdot a-30 \cdot b+c=0 \\ E q 2 & -40 \cdot a-20 \cdot b+c=-1\end{array}$

Eq $62-40 \cdot a+20 \cdot b+c=11$

Eq $63-40 \cdot a+30 \cdot b+c=12$

We will consider that this is a matrix type system $A \times N e c=B$

where: A - is the matrix with 3 columns and 63 lines of unknowns' coefficients; $N e c$ - is the column vector of the 3 unknowns ( $\mathrm{a}, \mathrm{b}, \mathrm{c})$; $\mathrm{B}$ - is the column vector of free terms.

The MATLAB program sequence required to solve this system is the following:

A1 = [-40 -30 1;-40 -20 1;-40 -10 1;-40 0 1;-40 10 1;-40 20 1;-40 301$]$

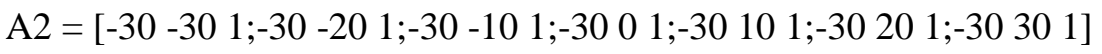

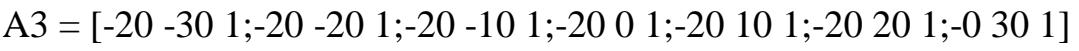

A4 = [-10 -30 1;-10 -20 1;-10 -10 1;-10 $01 ;-10101 ;-10201 ;-10301]$

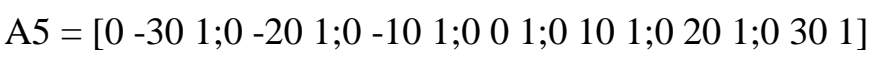

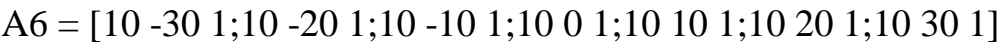

$\mathrm{A} 7=\left[\begin{array}{lllllllll}20 & -30 & 1 ; 20 & -20 & 1 ; 20 & -10 & 1 ; 20 & 0 & 1 ; 20 \\ 10 & 1 ; 20 & 20 & 1 ; 20 & 30 & 1\end{array}\right]$

$\mathrm{A} 8=\left[\begin{array}{lllllllll}30 & -30 & 1 ; 30 & -20 & 1 ; 30 & -10 & 1 ; 30 & 0 & 1 ; 30 \\ 10 & 1 ; 30 & 20 & 1 ; 30 & 30 & 1\end{array}\right]$

A9 = [40 -30 1;40-20 1;40-10 1;40 0 1;40 10 1;40 20 1;40 301$]$

$\mathrm{A}=[\mathrm{A} 1 ; \mathrm{A} 2 ; \mathrm{A} 3 ; \mathrm{A} 4 ; \mathrm{A} 5 ; \mathrm{A} 6 ; \mathrm{A} 7 ; \mathrm{A} 8 ; \mathrm{A} 9]$

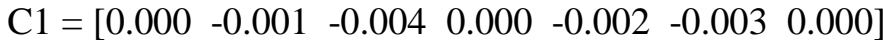

$\mathrm{C} 2=\left[\begin{array}{lllllll}0.003 & 0.004 & 0.006 & -0.002 & 0.007 & -0.005 & 0.005\end{array}\right]$

$\mathrm{C} 3=\left[\begin{array}{lllllll}0.006 & 0.003 & 0.009 & 0.005 & 0.009 & 0.008 & 0.009\end{array}\right]$

$\mathrm{C} 4=\left[\begin{array}{lllllll}0.004 & 0.007 & 0.008 & 0.009 & 0.010 & 0.012 & 0.012\end{array}\right]$

$\mathrm{C} 5=\left[\begin{array}{lllllll}0.005 & 0.009 & 0.009 & 0.008 & 0.011 & 0.013 & 0.011\end{array}\right]$

C6 $=\left[\begin{array}{lllllll}0.007 & 0.007 & 0.008 & 0.009 & 0.012 & 0.011 & 0.008\end{array}\right]$

$\mathrm{C} 7=\left[\begin{array}{lllllll}0.009 & 0.010 & 0.009 & 0.009 & 0.010 & 0.010 & 0.009\end{array}\right]$

$\mathrm{C} 8=\left[\begin{array}{lllllll}0.008 & 0.007 & 0.007 & 0.007 & 0.009 & 0.010 & 0.010\end{array}\right]$

$\mathrm{C} 9=\left[\begin{array}{lllllll}0.007 & 0.008 & 0.009 & 0.000 & 0.009 & 0.007 & 0.008\end{array}\right]$

$\mathrm{C}=\left[\begin{array}{lllllllll}\mathrm{C} 1 & \mathrm{C} 2 & \mathrm{C} 3 & \mathrm{C} 4 & \mathrm{C} 5 & \mathrm{C} 6 & \mathrm{C} 7 & \mathrm{C} 8 & \mathrm{C} 9\end{array}\right]$

$B=C^{\prime}$

$\mathrm{Nec}=\mathrm{A} / \mathrm{B} \Rightarrow \mathrm{Nec}=\left(\begin{array}{l}0.00009195002024 \\ 0.00003749164300 \\ 0.00664932104510\end{array}\right)$

Therefore, the equation of the wanted plane surface is:

$z=0.00009195 x+0.00003749 y+0.00664932$ 
Note 1: Since the matrix of coefficients is relatively large (3 columns and 63 lines), it is considered more convenient to write 9 matrices (A1, A2 ... A9) with 7 lines each in the first 9 lines of the program.

In the 10th line of the program, matrix A was performed by concatenating the A1 ... A9 matrices.

Note 2: Since the column matrix (free vector) is relatively large (63 terms), it is considered more convenient to write 9-line matrices $(\mathrm{C} 1, \mathrm{C} 2 \ldots \mathrm{C} 9)$ with 7 lines each in lines $11 \ldots 19$ of the program.

In the 20th line of the program, the $\mathrm{C}$-line matrix with 63 terms was created by concatenating the $\mathrm{C} 1$ ... C9 matrices.

The column matrix (column vector) B was obtained by transposing the matrix $\mathrm{C}$ line (line 21 of the program)

Note 3: In the last line of the program, the matrix of unknowns was calculated by using the matrix partitioning method, the MATLAB-specific partitioning method (Ghinea, 1995).

\section{The method of defining the plane surface by symmetric coordination}

In order to measure flatness deviations, the part under study can be mounted as in the case of the first method (the 3-random points method) or it can be placed directly on the control table. If the same measurement system is maintained then the same values of deviations (Table 1) will be obtained and these data will serve to determine the reference plane surface, defined by an equation of the type:

$\mathrm{z}=\mathrm{z}_{0}+\alpha \mathrm{x}+\beta \mathrm{y}$

where the free term $\mathrm{z} 0$ and the coefficients $\alpha$ and $\beta$ are given by the matrix system:

$\mathrm{z}_{\mathrm{i}}=\mathrm{z}_{0}+\alpha \mathrm{x}_{\mathrm{i}}+\beta \mathrm{y}_{\mathrm{i}} \quad(\mathrm{i}=1 \ldots \mathrm{n})$

In order to increase the accuracy of computation, the aim of the test must be to minimize rounding errors resulting from computational operations, by minimizing the number of algebraic operations, primarily those operations that inevitably lead to the rounding of numbers (division and multiplication operations).

The proposed solution consists in defining the origin of the Oxy coordinate system in the center of the piece and making a grid with symmetrical coordinates with respect to the Ox and Oy axes. Due to the symmetrically chosen coordinates on the Ox and Oy axes, the number of algebraic calculus operations can be greatly reduced and only a system of 3 equations with 3 unknowns will be solved.

First, the sum of the " $n$ " equations of the system 5 is obtained (where $n=$ the total number of measurement points - according to Figure 2 and Table 1, the result being $n=9 \times 7=63$ ), thus leading to a new equation:

$$
\begin{aligned}
& \sum_{\mathrm{i}=1}^{\mathrm{n}} \mathrm{z}_{\mathrm{i}}=\sum_{\mathrm{i}=1}^{\mathrm{n}} \mathrm{z}_{0}+\sum_{\mathrm{i}=1}^{\mathrm{n}} \alpha \cdot \mathrm{x}_{\mathrm{i}}+\sum_{\mathrm{i}=1}^{\mathrm{n}} \beta \cdot \mathrm{y}_{\mathrm{i}},(i=1 \ldots n) \\
& \sum_{\mathrm{i}=1}^{\mathrm{n}} \mathrm{z}_{\mathrm{i}}=\mathrm{n} \cdot \mathrm{z}_{0}+\alpha \cdot \sum_{\mathrm{i}=1}^{\mathrm{n}} \mathrm{x}_{\mathrm{i}}+\beta \cdot \sum_{\mathrm{i}=1}^{\mathrm{n}} \mathrm{y}_{\mathrm{i}},(i=1 \ldots n)
\end{aligned}
$$

Equation (6) will be the first equation of the final system.

The system equations (5) are then multiplied by $\mathrm{x}_{\mathrm{i}}$, resulting in the equivalent system:

$$
\mathrm{z}_{\mathrm{i}} \cdot \mathrm{x}_{\mathrm{i}}=\mathrm{z}_{0} \cdot \mathrm{x}_{\mathrm{i}}+\alpha \cdot \mathrm{x}_{\mathrm{i}}^{2}+\beta \cdot \mathrm{x}_{\mathrm{i} .} \cdot \mathrm{y}_{\mathrm{i}},(i=1 . . n)
$$

and then sum up the "n" equations of the equation system (7) resulting in a new equation:

$$
\begin{aligned}
& \sum_{\mathrm{i}=1}^{\mathrm{n}} \mathrm{z}_{\mathrm{i}} \cdot \mathrm{x}_{\mathrm{i}}=\sum_{\mathrm{i}=1}^{\mathrm{n}} \mathrm{z}_{0} \cdot \mathrm{x}_{\mathrm{i}}+\sum_{\mathrm{i}=1}^{\mathrm{n}} \alpha \cdot \mathrm{x}_{\mathrm{i}}^{2}+\sum_{\mathrm{i}=1}^{\mathrm{n}} \beta \cdot \mathrm{x}_{\mathrm{i} .} \cdot \mathrm{y}_{\mathrm{i}},(i=1 \ldots n) \\
& \sum_{\mathrm{i}=1}^{\mathrm{n}} \mathrm{z}_{\mathrm{i}} \cdot \mathrm{x}_{\mathrm{i}}=\mathrm{z}_{0} \cdot \sum_{\mathrm{i}=1}^{\mathrm{n}} \mathrm{x}_{\mathrm{i}} \cdot \mathrm{x}_{\mathrm{i}}+\alpha \cdot \sum_{\mathrm{i}=1}^{\mathrm{n}} \mathrm{x}_{\mathrm{i}}^{2}+\beta \cdot \sum_{\mathrm{i}=1}^{\mathrm{n}} \mathrm{x}_{\mathrm{i} .} \cdot \mathrm{y}_{\mathrm{i}},(i=1 \ldots n)(8)
\end{aligned}
$$

Equation (8) will be the second equation of the final system.

Then the system equations (5) are multiplied by $y_{i}$ resulting in the equivalent system: 
$\mathrm{z}_{\mathrm{i}} \cdot \mathrm{y}_{\mathrm{i}}=\mathrm{z}_{0} \cdot \mathrm{y}_{\mathrm{i}}+\alpha \cdot \mathrm{x}_{\mathrm{i}} \cdot \mathrm{y}_{\mathrm{i}}+\beta \cdot \mathrm{y}_{\mathrm{i}}^{2},(i=1 \ldots n)$

and then the "n" equations of the equation system (9) are summed up, resulting in a new equation:

$$
\begin{aligned}
& \sum_{\mathrm{i}=1}^{\mathrm{n}} \mathrm{z}_{\mathrm{i}} \cdot \mathrm{y}_{\mathrm{i}}=\sum_{\mathrm{i}=1}^{\mathrm{n}} \mathrm{z}_{0} \cdot \mathrm{y}_{\mathrm{i}}+\sum_{\mathrm{i}=1}^{\mathrm{n}} \alpha \cdot \mathrm{x}_{\mathrm{i}} \cdot \mathrm{y}_{\mathrm{i}}+\sum_{\mathrm{i}=1}^{\mathrm{n}} \beta \cdot \mathrm{y}_{\mathrm{i}}^{2},(i=1 \ldots n) \\
& \sum_{\mathrm{i}=1}^{\mathrm{n}} \mathrm{z}_{\mathrm{i}} \cdot \mathrm{y}_{\mathrm{i}}=\mathrm{z}_{0} \cdot \sum_{\mathrm{i}=1}^{\mathrm{n}} \mathrm{y}_{\mathrm{i}}+\alpha \cdot \sum_{\mathrm{i}=1}^{\mathrm{n}} \mathrm{x}_{\mathrm{i}} \cdot \mathrm{y}_{\mathrm{i}}+\beta \cdot \sum_{\mathrm{i}=1}^{\mathrm{n}} \mathrm{y}_{\mathrm{i}}^{2},(i=1 \ldots n)
\end{aligned}
$$

Equation (10) will be the third equation of the final system.

By bringing together equations (6), (8) and (10) the final system is obtained:

$$
\begin{aligned}
& \sum_{i=1}^{n} z_{i}=n \cdot z_{0}+\alpha \cdot \sum_{i=1}^{n} x_{i}+\beta \cdot \sum_{i=1}^{n} y_{i} \\
& \sum_{i=1}^{n} z_{i} \cdot x_{i}=z_{0} \cdot \sum_{i=1}^{n} x_{i} \cdot x_{i}+\alpha \cdot \sum_{i=1}^{n} x_{i}^{2}+\beta \cdot \sum_{i=1}^{n} x_{i} \cdot y_{i} \quad(i=1 \ldots n)(11) \\
& \sum_{i=1}^{n} z_{i} \cdot y_{i}=z_{0} \cdot \sum_{i=1}^{n} y_{i}+\alpha \cdot \sum_{i=1}^{n} x_{i} \cdot y_{i}+\beta \cdot \sum_{i=1}^{n} y_{i}^{2}
\end{aligned}
$$

The system (11) can be reordered according to the unknowns $\alpha, \beta$ and $\mathrm{z}_{0}$, as follows:

$$
\begin{aligned}
& \alpha \cdot \sum_{i=1}^{n} x_{i}+\beta \cdot \sum_{i=1}^{n} y_{i}+n \cdot z_{0}=\sum_{i=1}^{n} z_{i} \\
& \alpha \cdot \sum_{i=1}^{n} x_{i}^{2}+\beta \cdot \sum_{i=1}^{n} x_{i} \cdot y_{i}+z_{0} \cdot \sum_{i=1}^{n} x_{i}=\sum_{i=1}^{n} z_{i} \cdot x_{i} \\
& \alpha \cdot \sum_{i=1}^{n} x_{i} \cdot y_{i}+\beta \cdot \sum_{i=1}^{n} y_{i}^{2}+z_{0} \cdot \sum_{i=1}^{n} y_{i}=\sum_{i=1}^{n} z_{i} \cdot y_{i} \quad(i=1 . n)
\end{aligned}
$$

This system of 3 unknowns and 3 equations can be simplified because of the symmetry of the coordinates of the measurement points, giving the following result:

$$
\sum_{\mathrm{i}==}^{\mathrm{n}} \mathrm{x}_{\mathrm{i}}=0 ; \sum_{\mathrm{i}==}^{\mathrm{n}} \mathrm{y}_{\mathrm{i}}=0 ; \sum_{\mathrm{i}==}^{\mathrm{n}} \mathrm{x}_{\mathrm{i}} \cdot \mathrm{y}_{\mathrm{i}}=0,(i=1 . . n)
$$

The system (12) can be rewritten in the simplified form:

$$
\begin{aligned}
& \alpha \cdot 0+\beta \cdot 0+\mathrm{n} \cdot \mathrm{z}_{0}=\sum_{\mathrm{i}=1}^{\mathrm{n}} \mathrm{z}_{\mathrm{i}} \\
& \alpha \cdot \sum_{\mathrm{i}=1}^{\mathrm{n}} \mathrm{x}_{\mathrm{i}}^{2}+\beta \cdot 0+0 \cdot \mathrm{z}_{0}=\sum_{\mathrm{i}=1}^{\mathrm{n}} \mathrm{z}_{\mathrm{i}} \cdot \mathrm{x}_{\mathrm{i}} \quad \quad(\mathrm{i}=1 \ldots \mathrm{n}) \\
& \alpha \cdot 0+\beta \cdot \sum_{\mathrm{i}=1}^{\mathrm{n}} \mathrm{y}_{\mathrm{i}}^{2}+0 \cdot \mathrm{z}_{0}=\sum_{\mathrm{i}=1}^{\mathrm{n}} \mathrm{z}_{\mathrm{i}} \cdot \mathrm{y}_{\mathrm{i}}
\end{aligned}
$$

It can be noted that the system solution (14) is:

$$
\alpha=\frac{\sum_{i=1}^{n} z_{i} \cdot x_{i}}{\sum_{i=1}^{n} x_{i}^{2}}, \beta=\frac{\sum_{i=1}^{n} z_{i} \cdot y_{i}}{\sum_{i=1}^{n} y_{i}^{2}}, z_{0}=\frac{1}{n} \cdot \sum_{i=1}^{n} z_{i}
$$


Note 4: As shown by solution (14) the defining parameters of the reference plane surface can be determined by a minimal number of algebraic operations susceptible to rounding operations, and therefore the rounding errors are negligible if this method is applied.

Note 5: If, for equations (14), coordinates $\mathrm{x}_{\mathrm{i}}$ and $\mathrm{y}_{\mathrm{i}}$ are integers expressed in $\mathrm{mm}$ and $\mathrm{z}_{\mathrm{i}}$ deviations are expressed in $\mu \mathrm{m}$ or $\mathrm{mm}$ in the numerical calculation of the denominator and count values, there will be no rounding error.

By exemplifying the calculations for the data set in Table 1 it follows that:

$$
\begin{aligned}
& \sum_{i=1}^{63} z_{i}=487 \quad ; \quad \sum_{i=1}^{63} z_{i} \cdot x_{i}=5250 ; \sum_{i=1}^{63} z_{i} \cdot y_{i}=700 \\
& \sum_{i=1}^{63} x_{i}^{2}=42000 \quad ; \quad \sum_{i=1}^{63} y_{i}^{2}=19600
\end{aligned}
$$

and then, by making the required replacement in (15), the result is as follows:

$\alpha=3.86 / 42000=0.0000919$

$\beta=1.01 / 19600=0.0000515$

$\mathrm{z} 0=0.419 / 63=0.006651$

Therefore, the reference plane surface equation (П) will be:

$\mathrm{z}=0.0000919 \mathrm{x}+0.0000515 \mathrm{y}+0.00665$

or written in canonical form $\mathrm{Ax}+\mathrm{By}+\mathrm{Cz}+\mathrm{D}=0$, the reference plan equation becomes:

$0.0000919 \mathrm{x}+0.0000515 \mathrm{y}-\mathrm{z}+0.006651=0$

In the graphic representation of Fig. 3 this reference plan was noted with ( $\Pi$ ).

Note that, in the numerical example under focus, the equation obtained by the matrix solving of the equation system $(\mathrm{z}=0.00009195 \mathrm{x}+0.00003749 \mathrm{y}+0.00664932)$ is very similar to the equation obtained by the symmetrical coordinates method $(\mathrm{z}=0.0000919 \mathrm{x}+0.0000515 \mathrm{y}+0.006651)$.

Using the canonical form of the reference plane surface ( $\Pi$ ) in equation (18), the plane deviations can be calculated from the plane surface, using the formula of the distance from a point in space to a plane:

$a_{i}=\frac{A \cdot x_{i}+B \cdot y_{i}+C \cdot z_{i}+D}{\sqrt{A^{2}+B^{2}+C^{2}}}$

By applying this calculation formula for all couples $\left(\mathrm{x}_{\mathrm{i}}, \mathrm{y}_{\mathrm{i}}, \mathrm{z}_{\mathrm{i}}\right)$ in Table 1, the deviations from the plane defined by the smallest-squares method (table 2) were determined, as follows:

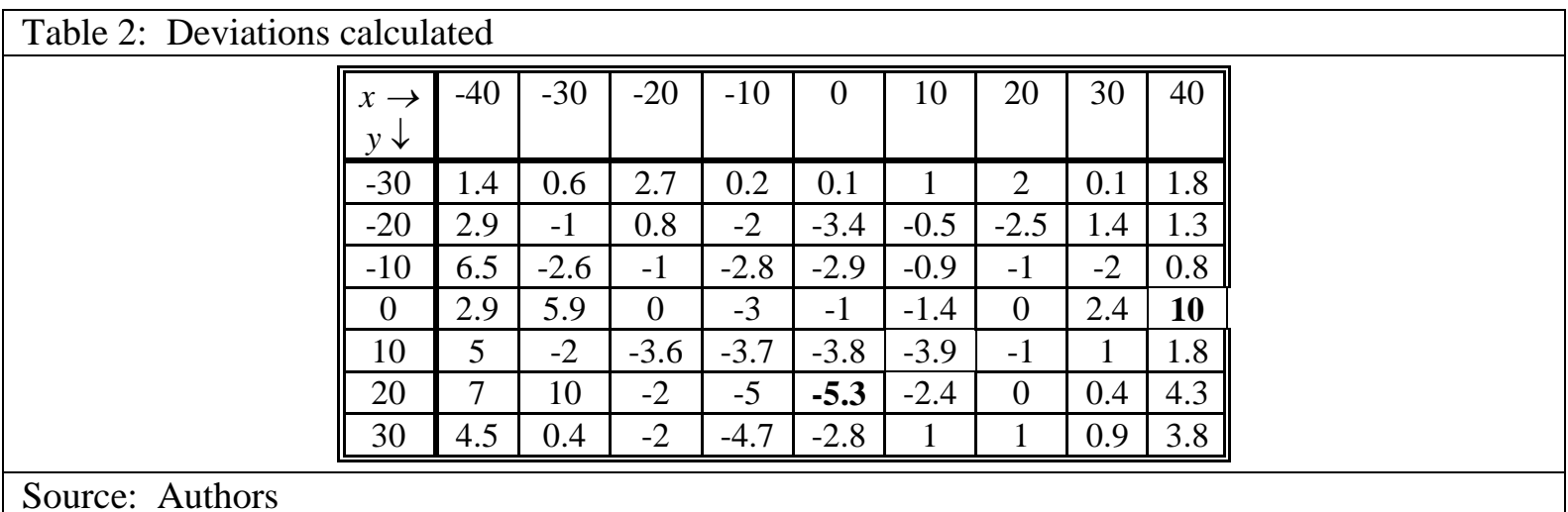

After a careful analysis of these data, it can be noted that the maximum flatness deviation after applying the symmetrical coordinates method is:

$\mathrm{AFp}_{2}=10+5.3=15.3 \mu \mathrm{m}<\mathrm{AFp}, 1=18 \mu \mathrm{m}<\mathrm{Tfp}=20 \mu \mathrm{m}$

\section{Conclusions}

As a result of those above presented, the method is simple and can be applied very quickly, it does not need calculations and it's very useful for parts with non-processed bearing surfaces. 
Disadvantages of the method: the reference plane surface is not the adjacent one, defined according to SR EN ISO 1101-2017; the reference plane surface is calculated with the help of three random points, therefore different measurements (made by manufacturers and beneficiaries respectively) may produce different results.

The calculation of the plane surface by the matrix (MATLAB) method is fairly accurate in identifying the reference plane surface, if we were to compare it to the three-random-points method; its accuracy increases with the number of points chosen.

Therefore, for the matrix-type solving of linear equation systems, the calculation of an " $\mathrm{n}$ " order determinant resulting from the use of minor determinants' development method implies $n$ ! multiplication operations and $n(n-1) / 2$ adding or subtraction operations. That is why the results may need a large number of rounding, thus influencing the accuracy of calculations.

The calculation method using symmetrical coordinates is advantageous because this method can be repeated successively and, if applied correctly by producers and beneficiaries, similar results can be achieved; even if a very high number of measurement points are used, the calculation method is simple, the number of algebraic operations is minimal and, as a result, rounding errors are insignificant. In the case of the numerical example given, solution (15) needs only one subtraction operation for which number rounding may be necessary; the method usually leads to minimal deviations compared to other methods (Bourget , 1996).

\section{References}

Bourget, J. M., (1996). Metrology - General Metrology Utility. Bayonne

Capitanu, L., Florescu, V., Badita, L.L (2016).High precision measurement technique of very small quantities of metallic wear, Romanian Review Precision Mechanics, Optics and Mechatronics , (50), p. 217-222.

Ghinea, M. et al, (1995), MATLAB - Numerical Calculus - Graphics - Applications. TEORA Publishing House, (Bucharest)

Legendi, A., (1994). Study of assembly geometry and stretching stress values for shale shaker grids. Scientific Journal of "Transilvania" University, (Brasov). Mechanics Supplement.

Rece, L. (1999). Mechanical technologies and control, Course. Volume I: Technical control. Multiplying shops at Conspress Publishing House, UTCB. Bucharest. 